\section{Nucleus Basalis of Meynert}

Tiffany L. Powell

Department of Neurosurgery, Virginia

Commonwealth University, Richmond, VA, USA

\section{Synonyms}

Basal nucleus of meynert; NBM

\section{Definition}

A major cholinergic pathway that houses the largest collection of cholinergic neurons is the brain.
The NBM is located in the deep gray and white matter that lies anterior to the thalamus and basal ganglia. The NBM contains the cell bodies of neurons that project cholinergic axons to the cerebral cortex. Cholinergic refers to the production, alteration, or release of acetylcholine. Acetylcholine, one of the first neurotransmitters to be discovered, is released from this nucleus and is used as a neurotransmitter in both the peripheral and central nervous systems. In the central nervous system acetylcholine functions to support memory, attention and learning. In Alzheimer's disease, the nucleus basalis of meynert is often degenerated; this may in part explain the memory loss so prominent in this condition. 